Author(s):

G. T. Garvey

Submitted to:

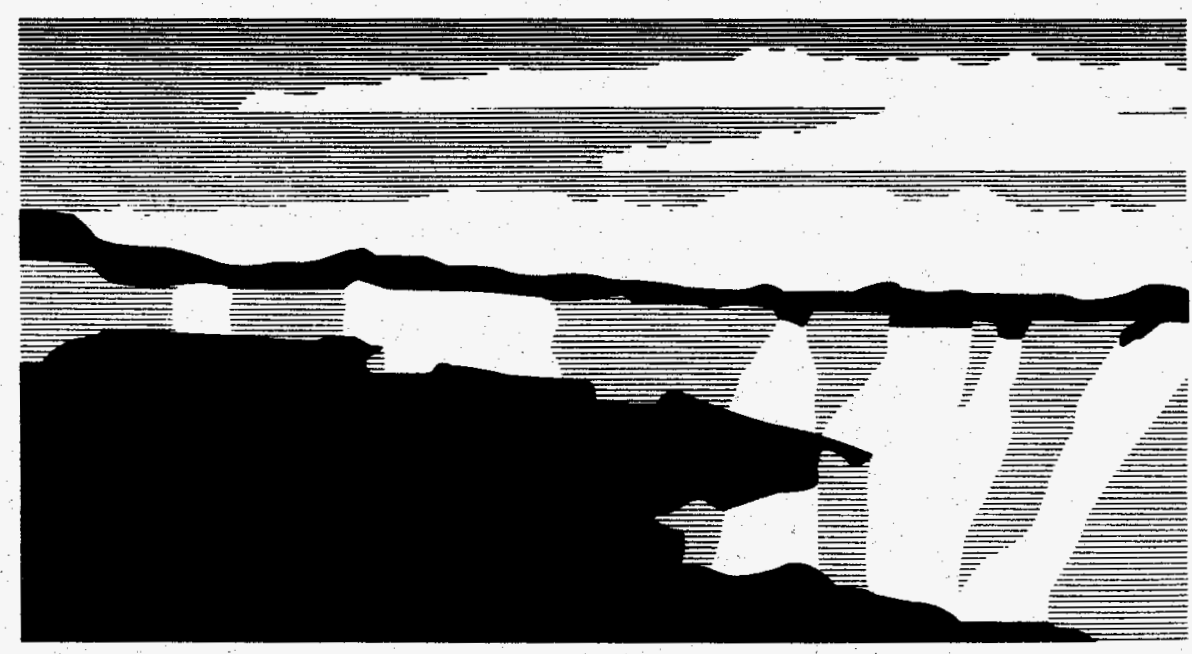

Los Alamos National Laboratory, an aftirmative action/equal opportunity employer, is operated by the University of California for the U.S. Department of Energy under contract W-7405-ENG-36. By acceptance of this article, the publisher recogrizes that the U.S. Government retains a nonexclusive, royalty-free license to publish or reproduce the published form of this contribution, or to allow others to do so, for U.S. Government purposes. The Los Alamos National Laboratory requests that the publisher identify this article as work performed under the auspices of the U.S. Department of Energy. 


\section{DISCLAIMER}

Portions of this document may be illegible in electronic image products. Images are produced from the best available original document. 


\title{
Neutral current neutrino-nucleon scattering
}

\author{
G. T. Garvey
}

Los Alamos National Laboratory, Los Alamos, NM 87545, USA

\section{INTRODUCTION}

It may appear unusual to have a contribution on neutrino scattering at a school devoted to electromagnetic probes, as the neutrino has no known electromagnetic couplings. However, as a means to examine the hidden flavor currents in the nucleon, the recent results [1] observed for the spin structure function of the nucleon have focused attention on the nucleon's neutral weak currents (NWC). When an electromagnetic probe is scattered elastically from a nucleon, the NWC interactions are observable only through the detection of very small $\left(>10^{-6}\right)$ parity-violating processes. In the case of neutrino scattering, the NWC is the dominant coupling.

In what follows it will be shown how the nucleon's vector and axial vector form factors arising from strange quark currents can be measured via neutrino elastic scattering. Preliminary results from the Large Scintillation Neutrino Detector (LSND) [2] at LAMPF will be presented as well as a recent analysis of an earlier experiment (E734) [3] carried out at Brookhaven.

\section{FORMALISM - FORM FACTORS}

The formalism will not be developed in full in the following. We will present only that portion required to provide a background to the subject and demonstrate how experimental results are used to obtain the required information.

It is well known to this group that the coupling of the proton vector current to a photon can be written

$$
\left\langle p^{\prime} s^{\prime}\left|J_{\mu}^{E m}\right| p, s\right\rangle=e \bar{\phi}_{p}\left(p^{\prime}, s^{\prime}\right)\left[F_{1}^{p}\left(Q^{2}\right) \gamma_{\mu}+F_{2}^{p}\left(Q^{2}\right) \frac{i \sigma_{\mu v} q_{v}}{2 M}\right] \phi_{p}(p, s)
$$

where $p$ and $s$ are the four momenta and spin of the initial state, etc., and $F_{1}^{P}\left(Q^{2}\right)$ and $F_{2}^{P}\left(Q^{2}\right)$ are the Dirac and Pauli form factors of the proton. In a more general representation using isospin and dropping the explicit $Q^{2}$ dependence in the form factors, 


$$
\left\langle p^{\prime}, s^{\prime}\left|J_{\mu}^{E m}\right| p, s\right\rangle=e \phi_{n}\left(p^{\prime}, s^{\prime}\right)\left(\left[F_{1}^{1}+F_{1}^{3} \tau_{3}\right] \gamma_{\mu}+\left[F_{2}^{1}+F_{2}^{3} \tau_{3}\right] \frac{i \sigma_{\mu v} p_{v}}{2 M}\right) \phi(p, s)
$$

where

$$
\begin{aligned}
& F_{i}^{1}=\frac{F_{1}^{p}+F_{1}^{n}}{2}, \\
& F_{i}^{3}=\frac{F_{1}^{p}-F_{1}^{n}}{2} .
\end{aligned}
$$

$\tau_{3}=+1$ for a proton and -1 for a neutron. The linear combination of form factors appearing in Eq. (3) is isoscalar while that in Eq. (4) is isovector. At $Q^{2}=0, F_{1}^{p}=1$ and $F_{1}^{n}=0$, while the anomalous magnetic moments at $Q^{2}=0$ are $F_{2}^{P}=1.7928$ and $F_{2}^{n}=-1.9130$.

These form factors arise from the underlying quark currents in the nucleon, which are written

$$
\left\langle p^{\prime}, s^{\prime}\left|J_{\mu}^{E m}\right| p, s\right\rangle=e \bar{\phi}_{n}\left(p^{\prime}, s^{\prime}\right)\left(\sum_{i} Q_{i} \bar{q}_{i} \gamma_{\mu} q_{i}\right) \phi_{n}(p, s)
$$

where $Q_{i}$ is the charge on quark flavor $i$.

To high accuracy the sum over quark flavors, $i$, need only extend over $u, d$, and $s$. Thus,

$$
\left\langle p^{\prime}, s^{\prime}\left|J_{\mu}^{E m}\right| p, s\right\rangle=e \bar{\phi}_{n}\left(p^{\prime}, s^{\prime}\right)\left[\frac{2}{3} \bar{u} \gamma_{\mu} u-\frac{1}{3} \bar{d} \gamma_{\mu} d-\frac{1}{3} \bar{s} \gamma_{\mu} s\right] \phi_{n}(p, s),
$$

or, rewriting in an isospin representation,

$$
\left\langle p^{\prime}, s^{\prime}\left|J_{\mu}^{E m}\right| p, s\right\rangle=e \bar{\phi}_{n}\left(p^{\prime}, s^{\prime}\right)\left[\frac{1}{2}\left(\bar{u} \gamma_{\mu} u-\bar{d} \gamma_{\mu} d\right)+\frac{1}{6}\left(\bar{u} \gamma_{\mu} u+\bar{d} \gamma_{\mu} d-2 \bar{s} \gamma_{\mu} s\right)\right] \phi_{n}(p, s)
$$

The first term on the right is clearly the third component of the isovector vector current in the nucleon. The second term is the isoscalar current. Recall that only $u$ and $d$ quarks carry strong isospin. Of course; at this time, these currents cannot be calculated because nonperturbative $Q C D$ is not yet implemented. However, it will be seen that by using this representation for the currents, it is straightforward to relate the form factors that characterize the nucleon coupling to the various gauge bosons $\left(\gamma, W^{ \pm}, Z^{0}\right)$.

Consider the coupling of the nucleon to the charged gauge boson $W^{ \pm}$for $Q^{2} \ll M_{w}^{2}$, which is written

$$
\left\langle p^{\prime}, s^{\prime}\left|J_{\mu}^{W^{ \pm}}\right| p, s\right\rangle=\sqrt{\frac{G_{F}}{\sqrt{2}}} \bar{\phi}\left(p^{\prime}, s^{\prime}\right)\left[G_{A}^{3} \gamma_{\mu} \gamma^{5}+G_{P}^{3} \frac{q_{\mu} \gamma^{5}}{2 M}+F_{1}^{3} \gamma_{\mu}+F_{2}^{2} \frac{i \sigma_{\mu v} q_{v}}{2 M}\right] \tau^{ \pm} \phi_{n}(p, s),
$$

where the explicit $Q^{2}$ dependence of the form factor has again been suppressed. $G_{A}^{3}$ and $G_{P}^{3}$ are the axial vector and induced pseudoscalar form factors of the nucleon. At $Q^{2}=0, G_{1}^{3}=1.256 \pm 0.003$ and $G_{P}^{3}$ does not enter into any of the cases of interest to us. The vector form factors can, of course, be related to the 
corresponding electromagnetic form factors via CVC. The form factors in Eq. (8) are all necessarily isovector because the process is charge changing. Expressing the weak charge-changing currents in terms of the underlying quark currents, one has

$$
\left\langle p^{\prime}, s^{\prime}\left|J_{\mu}^{W^{ \pm}}\right| p, s\right\rangle=\sqrt{\frac{G_{F}}{\sqrt{2}}} \bar{\phi}_{n}\left(p^{\prime}, s^{\prime}\right)\left(\sum_{i} \bar{q}_{i} \gamma_{\mu}\left(1-\gamma^{5}\right) t^{ \pm} q_{i}\right) \phi_{n}(p, s)
$$

As the initial and final state must be a nucleon, the only quark flavors $(i)$ that can enter the sum over $i$ are $u$ and $d$. Charge changing involving any other flavor would produce a nucleon in the final state. Written in this form, it is immediately evident that the vector current in Eq. (9) is the isovector nucleon form factor of Eq. (4) obtained from the coupling of nucleons to the photon (CVC).

The nucleon's coupling [4-8] to the $Z^{0}$ is specified by three form factors for $Q^{2} \ll M_{z}^{2}$,

$$
\left\langle p^{\prime}, s^{\prime}\left|J_{\mu}^{z^{0}}\right| p, s\right\rangle=\sqrt{\frac{G_{F}}{\sqrt{2}}} \bar{\phi}_{n}\left(p^{\prime}, s^{\prime}\right)\left[G_{A}^{z} \gamma_{\mu} \gamma^{5}+F_{1}^{z} \gamma_{\mu}+F_{2}^{z} \frac{i \sigma_{\mu \nu} p_{v}}{2 M}\right] \phi_{n}(p, s)
$$

In terms of quark currents, Eq. (10) is written as

$$
\left\langle p^{\prime}, s^{\prime}\left|J_{\mu}^{Z^{0}}\right| p, s\right\rangle=\sqrt{\frac{G_{F}}{\sqrt{2}}} \bar{\phi}_{n}\left(p^{\prime}, s^{\prime}\right)\left(\sum_{i} \bar{q}_{i} \gamma_{\mu}\left(1-\gamma^{5}\right) t_{z} q_{i}-2 Q_{i} \sin ^{2} \theta_{\omega} \bar{q}_{i} \gamma_{\mu} q_{i}\right) \phi_{n}(p, s)
$$

The first term on the right is a quark current identical to the charge-changing coupling to the $W^{ \pm}$, while the second term is an additional vector current due to the photon admixture in the $Z^{0}$. However, in both terms the sum over flavors is no longer restricted to only up and down quarks, as a quark need not reveal its flavor in neutral current scattering. It has been shown [4] that it is sufficiently accurate (2\%) for our purposes to restrict the sum over $u, d$, and $s$. It is straightforward using Eqs. (2) and (7)-(11) to write

$$
\begin{aligned}
& G_{A}^{Z^{0}}=-\frac{G_{A}^{3} \tau_{z}}{2}+\frac{G_{A}^{s}}{2}, \\
& F_{1}^{Z^{0}}=\left(1-2 \sin ^{2} \theta_{W}\right) F_{1}^{3} \tau_{z}-2 \sin ^{2} \theta_{W} F_{1}^{1}-\frac{F_{1}^{s}}{2}, \\
& F_{2}^{Z^{0}}=\left(1-2 \sin ^{2} \theta_{W}\right) F_{2}^{3} \tau_{z}-2 \sin ^{2} \theta_{W} F_{2}^{3}-\frac{F_{2}^{s}}{2},
\end{aligned}
$$

where

$$
\bar{\phi}_{n}\left(p^{\prime}, s^{\prime}\right) G_{A}^{s} \gamma_{\mu} \gamma^{5} \phi_{n}(p, s)=\bar{\phi}_{n}\left(p^{\prime}, s^{\prime}\right) \bar{s} \gamma_{\mu} \gamma^{5} \phi_{n}(p, s)
$$

etc. The nonrelativistic reduction of $\phi\left(p^{\prime}, s^{\prime}\right) \gamma_{\mu} \gamma^{5} \phi(p, s)$ is $\phi\left(p, s^{\prime}\right) \sigma \phi(p, s)$, so that $G_{A}^{s}$ is a measure of the strangeness contribution to the nucleon's spin, which accounts fõr its great contemporary interest. It provides a totally independent way to measure the strangeness contribution to the nucleon's spin. It avoids the difficulties of higher twist contributions, extrapolation to $x \rightarrow 0$, and assumptions of SU(3) symmetry, all of which are encountered in extracting the same information from the spin structure functions. Though much 
simpler in concept than polarized deep inelastic scattering, a precision measurement of neutrino-nucleon scattering is formidable.

\section{NEUTRINO-NUCLEON CROSS SECTION}

In terms of these form factors, the complete expression $[3,9,10]$ for neutrino-nucleon scattering is written

$$
\frac{d \sigma^{\frac{v}{v}}}{d \Omega}=\frac{G_{F}^{2}}{2 \pi} \frac{Q^{2}}{E_{v}^{2}}\left[A \pm B \omega+C \omega^{2}\right]
$$

where

$$
\omega \equiv \frac{4 E_{v}}{M}-\frac{Q^{2}}{M^{2}}, \quad \tau \equiv \frac{Q^{2}}{4 M^{2}},
$$

and

$$
\begin{aligned}
& A=\frac{1}{4}\left[G_{A}^{Z^{0^{2}}}(1+\tau)-\left(F_{1}^{Z^{0^{2}}}-\tau F_{2}^{Z^{02}}\right)(1-\tau)+4 \tau F_{1}^{Z^{0}} F_{2}^{Z^{0}}\right] \\
& B=\frac{1}{4} G_{A}^{Z^{0}}\left(F_{1}^{Z^{0}}+F_{2}^{Z^{0}}\right) \\
& C=\frac{1}{16} \frac{M^{2}}{Q^{2}}\left[G_{A}^{Z^{02}}+F_{1}^{Z^{02}}+\tau F_{2}^{Z^{02}}\right] .
\end{aligned}
$$

Thus, by referring to Eq. (13), one can see that the neutrino-nucleon electron scattering cross section is specified in terms of known kinematics and known form factors apart from the strange form factors $F_{1}^{s}, F_{2}^{s}$, and $G_{A}^{s}$. These strange form factors can be sorted out via their very different $Q^{2}$ and via interference effects explicitly shown in Eq. (14). A discussion of the $Q^{2}$ dependence of the form factors is found in Refs. [10] and [11].

At low $Q^{2}, Q^{2} \ll M^{2}$, the cross section is essentially independent of $Q^{2}$ and dominated by the value of $G_{A}^{Z^{0}}(0)$. Figure 1 shows the predicted cross sections for $v-p$ and $v-n$ scattering, both for $G_{A}^{s}(0)=0$ and $G_{A}^{s}(0)=-0.19$. The latter is the value extracted by the EMC group based on measurement of the proton spin structure function. This value of $G_{A}^{s}$ has a $25 \%$ effect on the yield, increasing the proton cross section and decreasing the neutron cross section because of the explicit isospin dependence evidenced in Eq. (12a). The effects of $G_{A}^{s}(0)$ on the cross sections are shown explicitly in Figs. 1 and 2.

\section{EXPERIMENTAL CONSIDERATIONS}

The only observable in neutrino-nucleon scattering is the recoil momentum of the struck nucleon. The kinetic energy of the recoiling nucleon is given by 


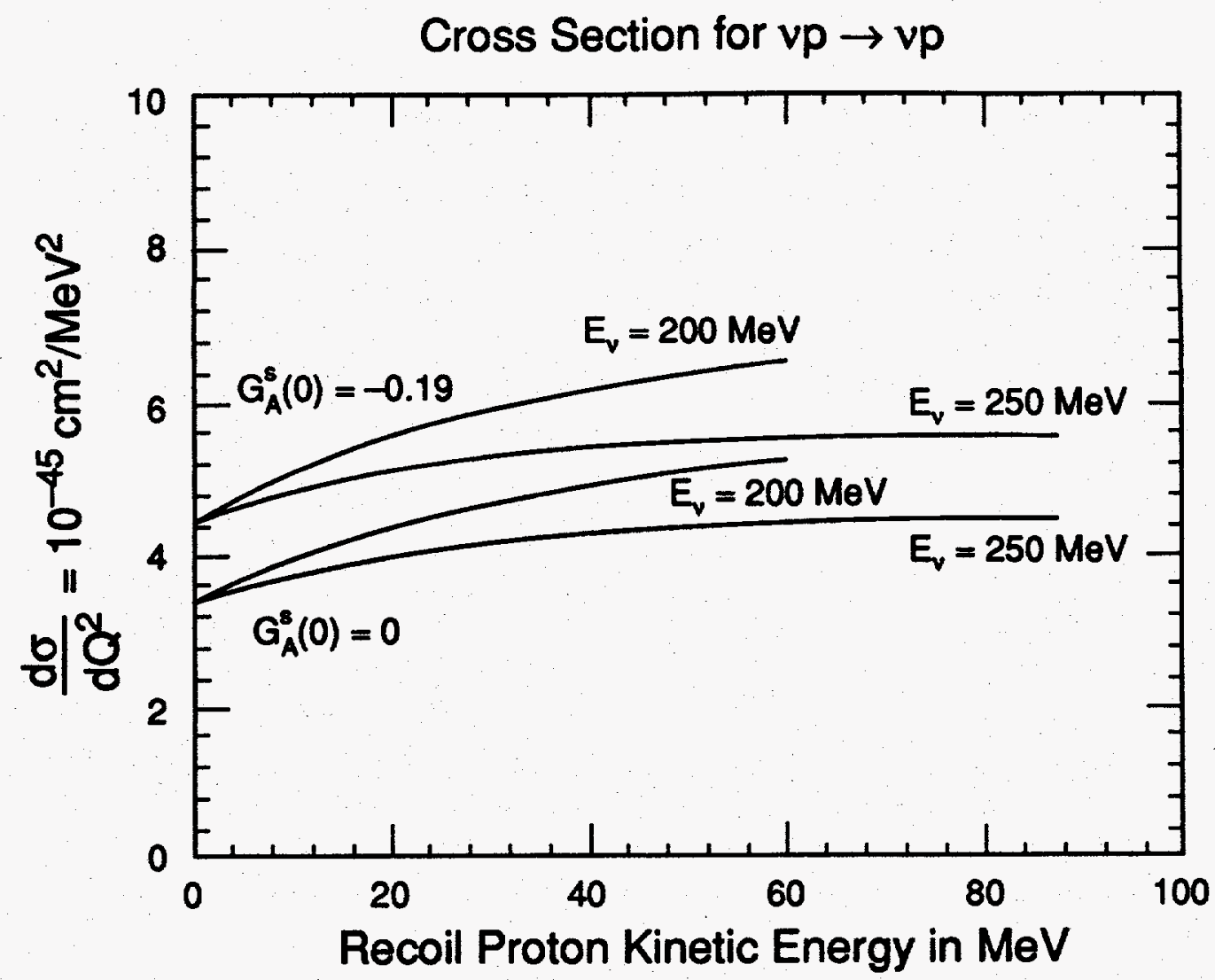

Fig. 1. Cross sections for neutral-current neutrino-proton elastic scattering at different incident neutrino energies. The plots show the effect of including a strange axial vector contribution $G_{s}(0)$.

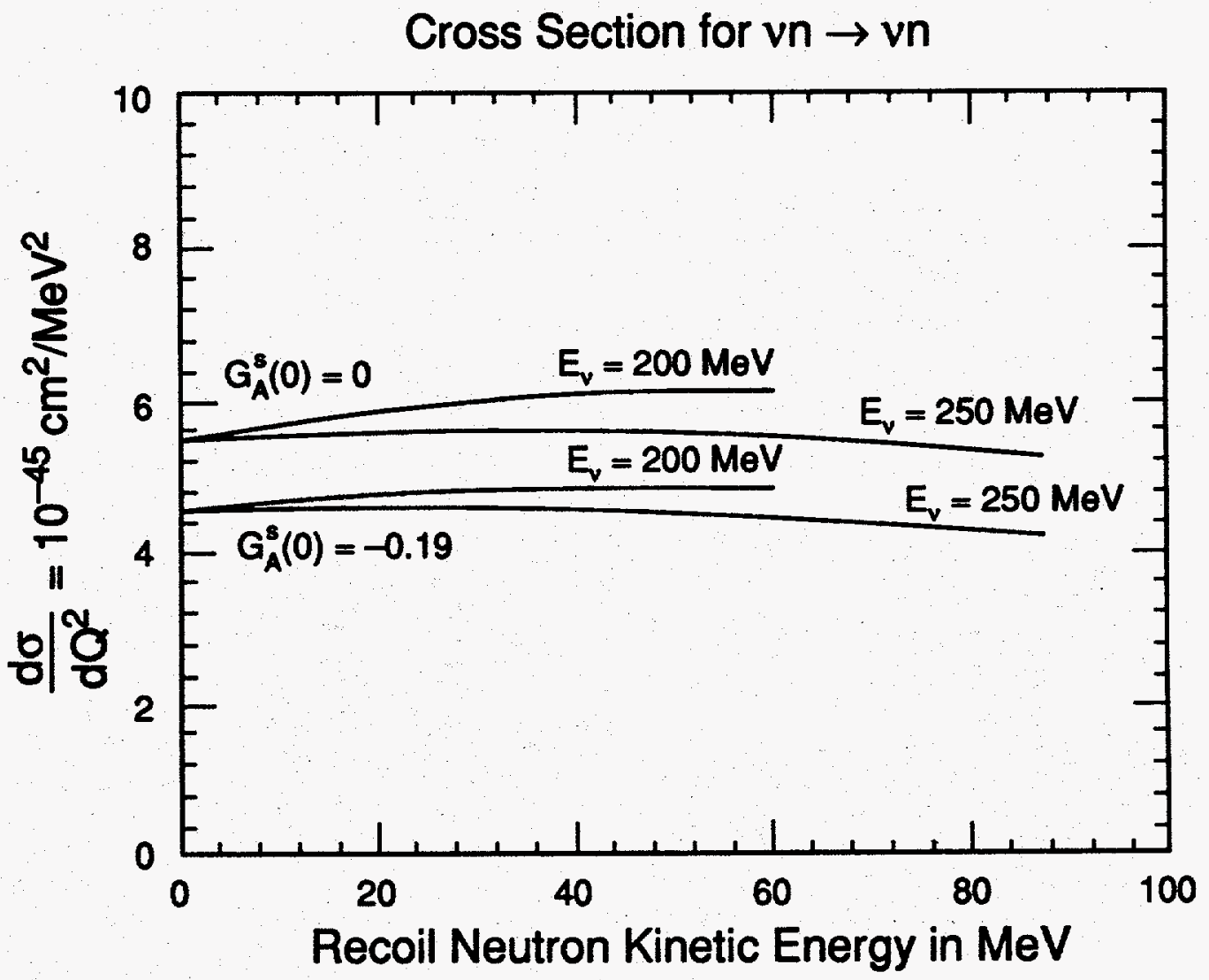

Fig. 2. Same as Fig. 1 but for neutrino-neutron elastic scattering. 


$$
T_{N}=\frac{Q^{2}}{2 M}=\frac{\left(2 E_{v} \sin ^{2} \frac{\theta}{2}\right)^{2}}{2 M_{p}\left(1+\frac{2 E_{v}}{M_{p}} \sin ^{2} \frac{\theta}{2}\right)}
$$

Thus, neutrinos of $100 \mathrm{MeV}$ or more must be employed to yield detectable signals $\left(T_{N}>20 \mathrm{MeV}\right)$ in the large detectors (100 tons) required to yield a useful number of events. Thus, neutrinos that originate from decay of mesons in flight are required.

A direct, precise (5\%) absolute measurement of neutrino-proton elastic scattering is difficult because it requires a precise knowledge of the neutrino flux, and it is generally not possible to separate scattering off free protons from those bound in the other nuclei typically present in the detector. An alternate procedure is to measure the ratio of proton-to-neutron yield from isoscalar $(N=Z)$ nuclei present in the detector. For light $(Z \leq 6)$ nuclei, the bound states of neutrons and protons are identical to high accuracy $(<1 \%)$, and as long as sufficiently energetic nucleons are detected, the effects of the Coulomb field on the final states are small and readily treated.

In the example presented below, the detector (LSND) consists of mineral oil $\left(\mathrm{CH}_{2}\right)$ to better than $10^{-3}$. The ${ }^{12} \mathrm{C}$ nuclei in such a detector are an example of an isoscalar target, and by selecting nucleons with kinetic energy above $60 \mathrm{MeV}$, contributions from the $\mathrm{H}$ nuclei can be avoided. The nucleons from $v$-C scattering can have higher final kinetic energy than free $v-p$ scattering because of the Fermi momentum in the initial bound state.

Figure 3 shows a calculation of the ratio of neutron-to-proton yield from $200-\mathrm{MeV}$ neutrinos on ${ }^{12} \mathrm{C}$ from Ref. [12]. In the absence of a strangeness contribution to the form factors, the ratio of proton-to-neutron yield

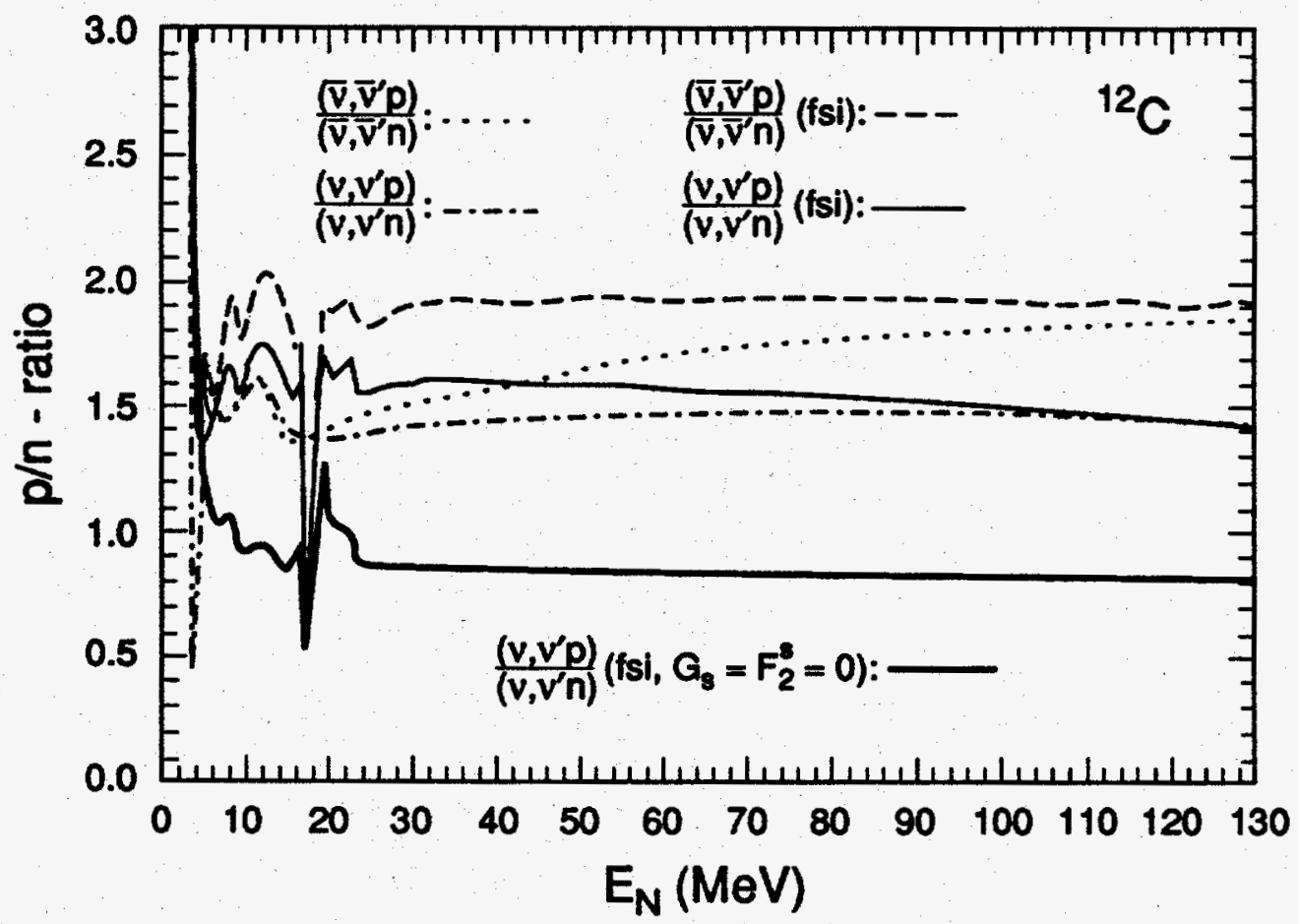

Fig. 3. Ratio of the $\left(v, v^{\prime} p\right)$ to $\left(v, v^{\prime} n\right)$ yield from $v^{12} \mathrm{C}$ as a function of $E_{N}=E_{p}=E_{n}+$ $2.2 \mathrm{MeV}$. Taken from Ref. [12], the ratios are shown for the $G_{s}(0)=0$ (lower curve) and for $G_{s}(0)=-0.19$ (upper four curves). 


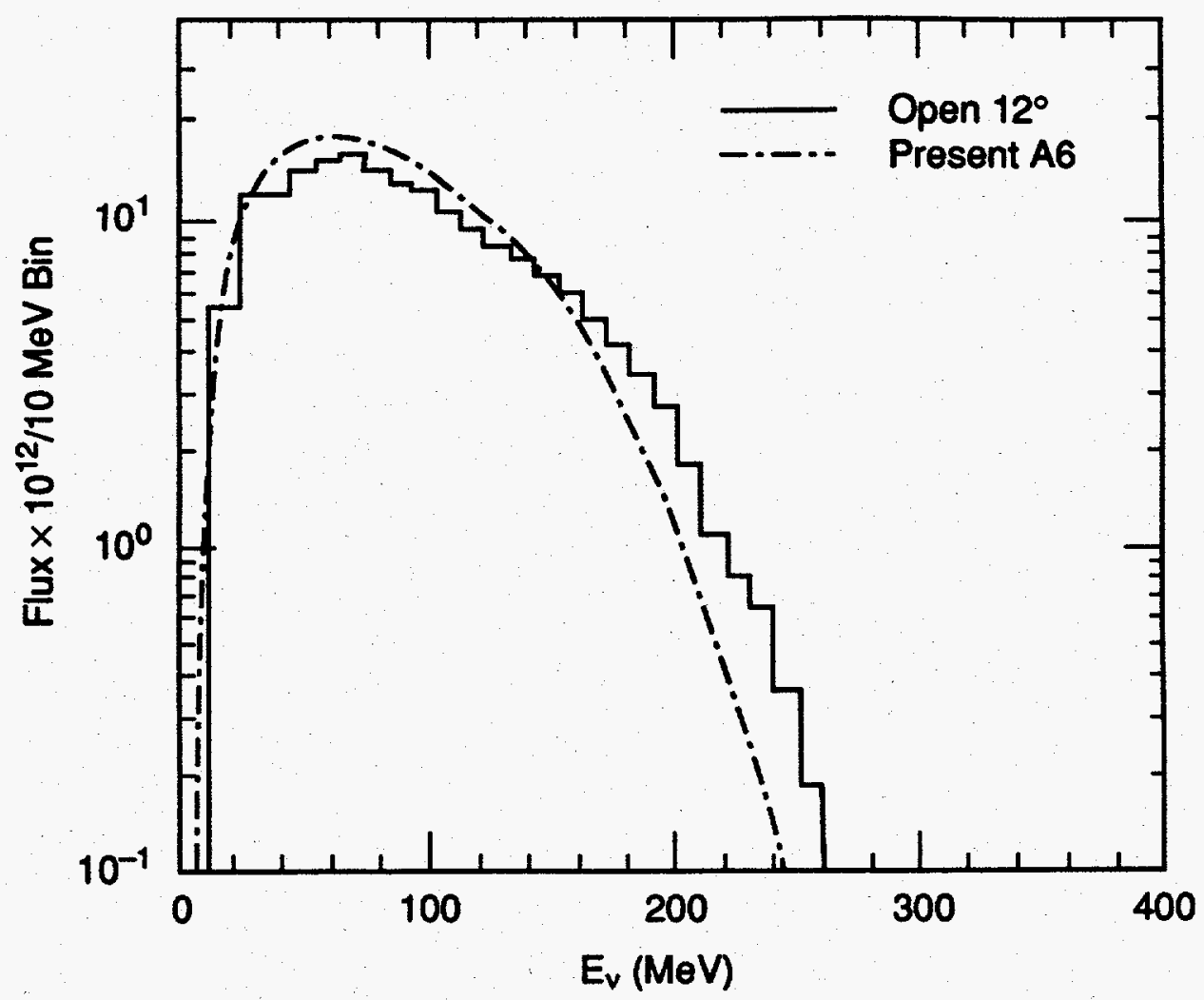

Fig. 4. Neutrino flux at LAMPF.

is 0.88 however. With $G_{A}^{s}=-0.19$, the ratio becomes 1.45 . In a very different calculation carried out in Ref. [13] at $E_{v}=150 \mathrm{MeV}$, very similar results are found. Thus, the ratio of neutron-to-proton yield from an isoscalar target is very sensitive to $G_{A}^{s}$ and insensitive to details of the neutrino flux. This effect is reflected in Figs. 1 and 2 where the dependence of free neutron and proton cross sections is sensitive to the neutral current coupling to each nucleon type. While final-state interactions might be a concern, the calculations of Ref. [12] show them to be a small correction.

Using the $v_{\mu}$ spectrum of Fig. 4 and accounting for the $\bar{v}_{\mu}$ s that are present in the LAMPF decay-in-flight beam produces the result shown in Fig. 5. Because the momentum transfer must be higher $\left(Q^{2}>0.11 \mathrm{GeV}\right)$ to obtain $E_{n}>60 \mathrm{MeV}$, there is some sensitivity of the proton-to-neutron yield to $F_{2}^{s}(0)$. Figure 5 shows the neutron-to-proton yield, reflecting the maximum expected range of $F_{2}^{s}(0)$. However, it is likely that the value of $F_{2}^{s}(0)$ is small, and at any rate it will soon be measured. If the value of $F_{2}^{s}(0)$ is taken as known, then a $10 \%$ measurement of the proton-to-neutron yield fixes the value of $G_{s}(0)$ to \pm 0.035 , an attractively small uncertainty at this stage of our knowledge of strangeness contributions to the nucleon spin.

\section{THE LSND EXPERIMENT}

Pions produced by the 800-MeV LAMPF proton beam produce the decay-in-flight neutrino flux shown in Fig. 4 at a distance of $25.5 \mathrm{~m}$ from the production target. The production target is at the LAMPF beam stop, so these neutrinos are available whenever the accelerator is operating. As follows from Eq. (15), neutrinos of more than $160 \mathrm{MeV}$ are required to produce recoiling nucleons with kinetic energy above $50 \mathrm{MeV}$. Thus, 


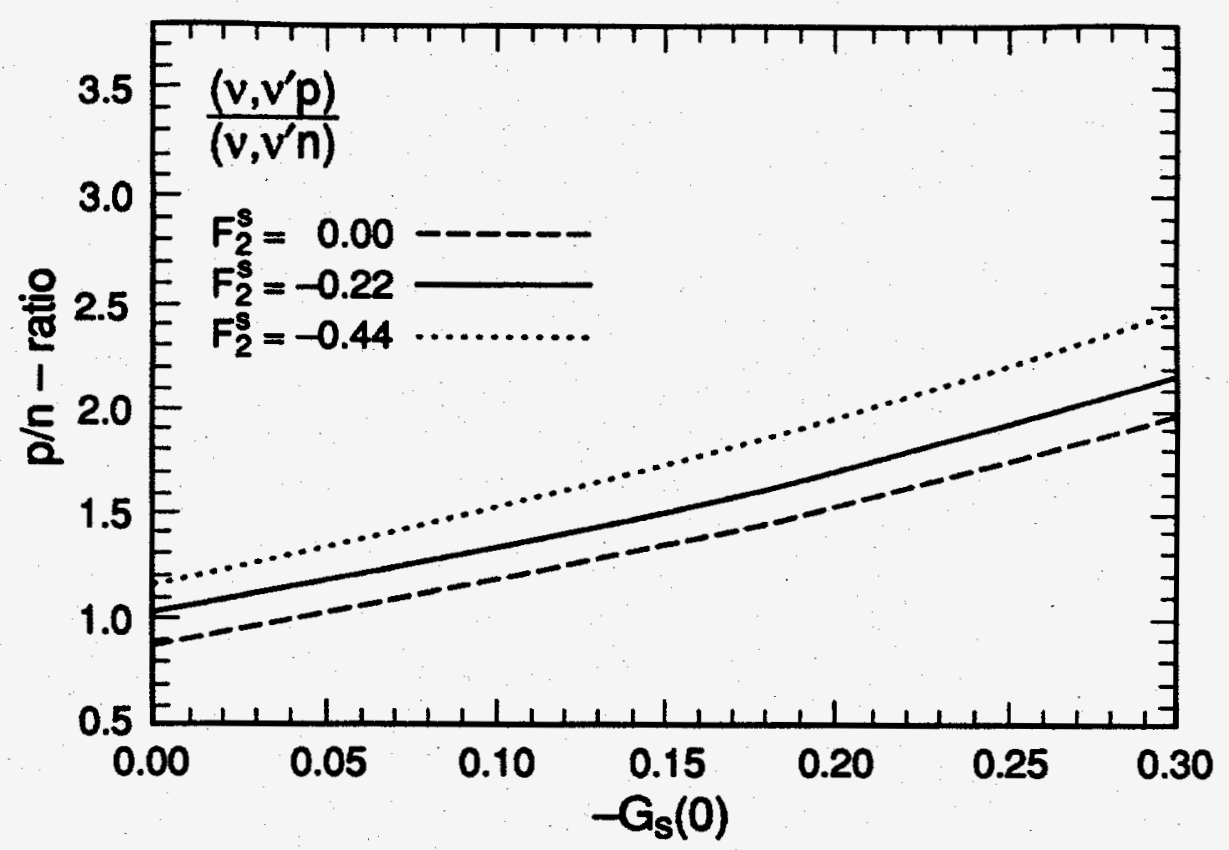

Fig. 5. Predicted values of the $v p / v n$ ratio for different values of $F_{2}^{S}$ as a function of $G_{s}$.

only the upper end of the $v$ spectrum shown in Fig. 4 is relevant to the following discussion of neutrinonucleon scattering.

LSND is a $v$ detector employing 200 tons of mineral oil $\left(\mathrm{CH}_{2}\right)$ supplemented by the addition of a small amount of scintillator $(0.03 \mathrm{~g} / \ell$ Butyl PPD). This mixture [14] permits the detection of scintillation light without obscuring the Cerenkov ring produced by highly relativistic $(v / c>0.66)$ particles. The $124020.3-c$ diameter Hamamatsu PMTs provide 25\% coverage, which yields 31 PEs per MeV of electron loss and 10 PEs per MeV for protons. For a highly relativistic particle, some $25 \%$ of the light is in the Čerenkov ring.

Figure 6 shows a diagram of the detector, which is $8.75 \mathrm{~m}$ in length and $5.72 \mathrm{~m}$ in diameter. An elaborate data acquisition system records the individual PM charge and time of arrival of the initial photoelectron (to $0.8 \mathrm{~ns}$ precision/tube) every $100 \mathrm{~ns}$. A continuously updating buffer retains these data for $200 \mu \mathrm{s}$, which is selectively accessed when the detector trigger is activated. Upon being triggered, the previous $52-\mu$ s history of all tubes above their discrimination threshold is presented along with the event itself. The triggering system also allows a 1-ms search for subsequent low-energy ( $>0.6 \mathrm{MeV}$ ) activity in the detector following specified trigger levels.

The detector is placed within a highly efficient veto shield that was designed and used in an earlier experiment. The shield is operated in an anti-coincidence mode with the trigger and imposes a 15- $\mu$ s dead time on the trigger to suppress the high rate that would otherwise occur from the electron decay of CR muons that stop in the detector (23\%). A complete 50- $\mu$ s history of the $300 \mathrm{PM}$ tubes in the veto shield is also obtained with each triggered event.

Figure 7 shows the relative number of events recorded as a function of the number of hit tubes. The events shown construct as being at least $50 \mathrm{~cm}$ inside the volume defined by the PMT faces. The trigger level was set at 100 hit tubes; however, when the number of hit tubes exceeds 300 , the trigger threshold is dropped to 18 hit tubes for the subsequent $1 \mathrm{~ms}$. The overall trigger rate is approximately $35 \mathrm{H}$. The peak just beyond 100 hit tubes is due to ${ }^{12} \mathrm{~B}$ beta decay. The ${ }^{12} \mathrm{~B}$ is produced via $\mu^{-}+{ }^{12} \mathrm{C} \rightarrow v_{\mu}+{ }^{12} \mathrm{~B}$. The events between 200-500 hits are, for the most part, Michel electrons from the decay of stopped CR muons that have not yet 

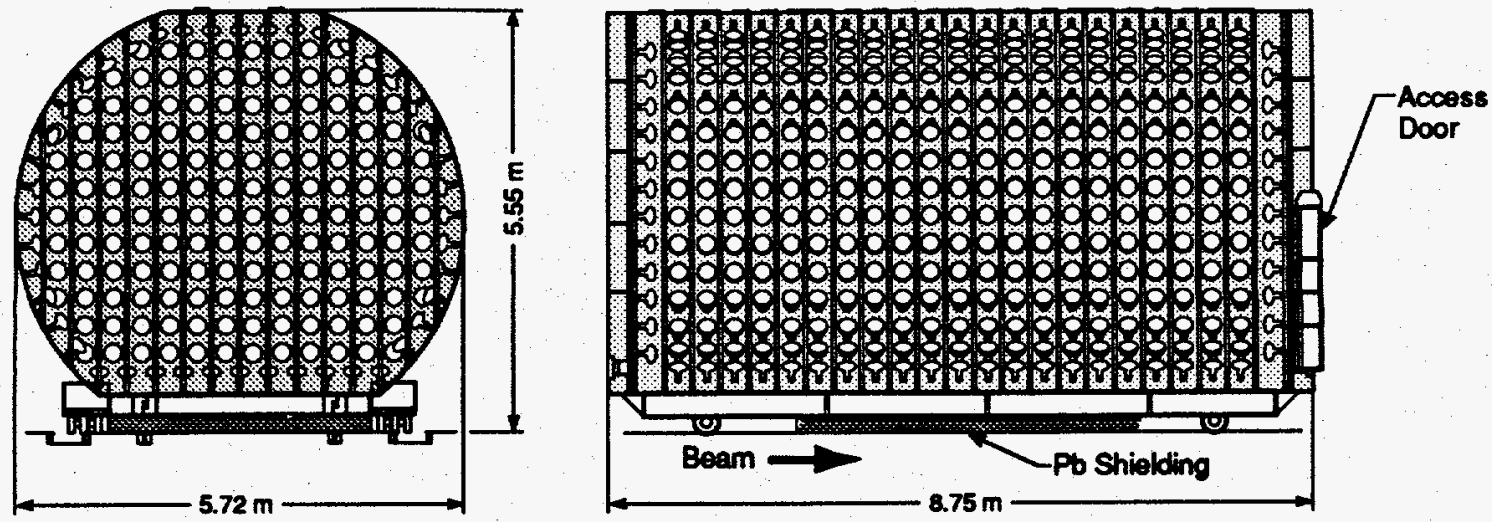

Fig. 6. Diagram of the LSND.

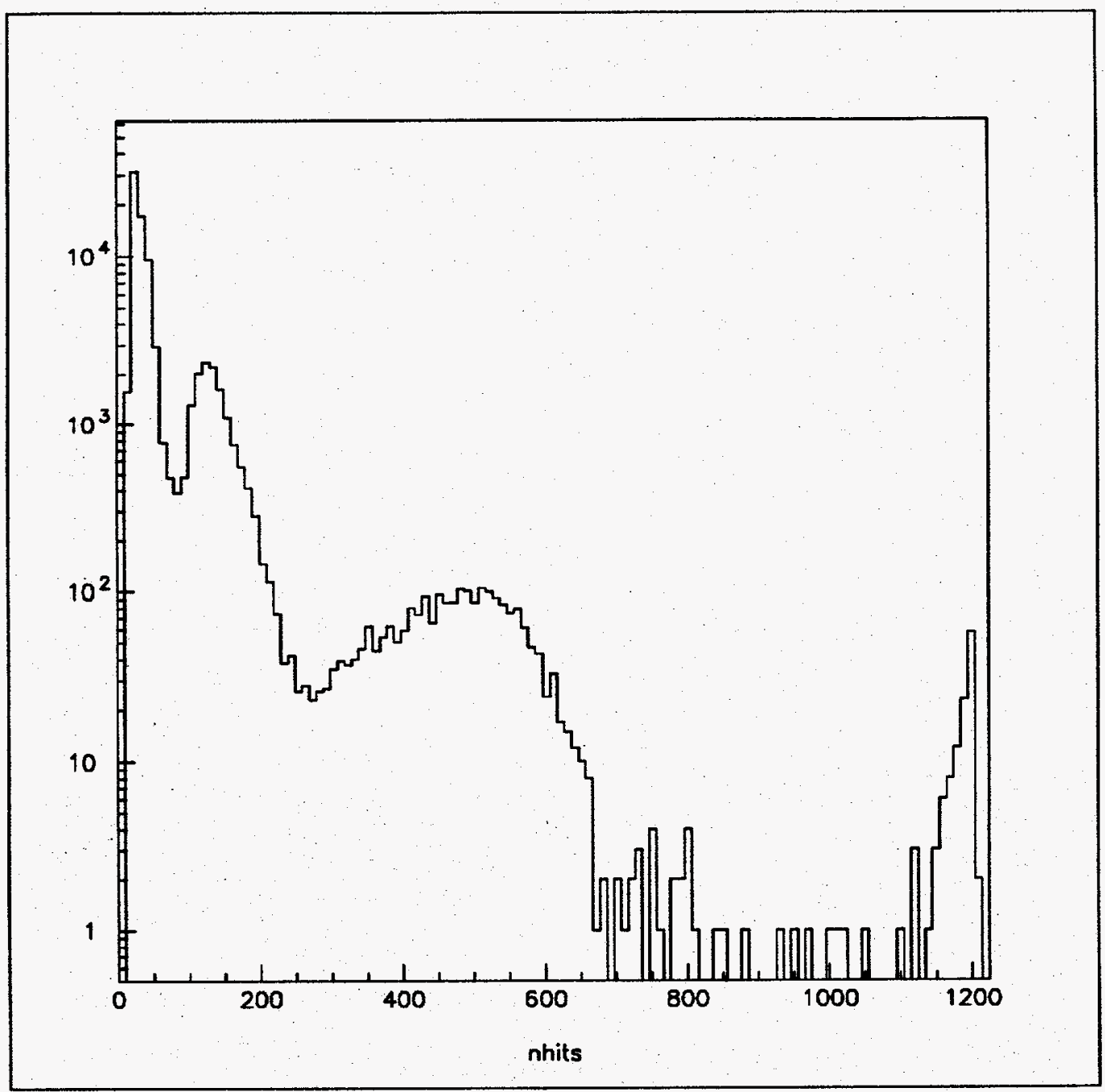

Fig. 7. The events recorded in the LSND detector as a function of the number of hit tubes. The detector trigger level is set at $\mathbf{1 0 0}$ hit tubes. When the number of hit tubes exceeds 300 , the trigger level drops to 18 hit tubes for $1 \mathrm{~ms}$. The rate of Michel electrons is suppressed by a factor of $7 \times 10^{4}$ by the $15.2-\mu \mathrm{sec}$ dead time imposed by triggering the veto shield.

decayed after the 15.2- $\mu \mathrm{s}$ CR veto signal. The events out beyond 1000 hit tubes are CR muons that slip through the first level veto ( $>6$ hit tubes in the veto counter). The events below channel 100 are those that occur in the $1 \mathrm{~ms}$ following events that fired more than 300 tubes. 
Figure 7 shows the remarkable dynamic range that has been achieved with LSND. Cosmic-ray muons deposit up to $2 \mathrm{GeV}$ in the detector, while $\gamma$ rays as low as $0.6 \mathrm{MeV}$ are detected in the triggered 1-ms interval.

LSND is capable of particle identification because of its sensitivity to Cerenkov light, which in addition to having a directional correlation in the associated light, yields a smaller fraction of late light $(t>20 \mathrm{~ns})$ than is the case for light from the scintillation process. The ID signal associated with an event is generated by a threefold product of the variance in an event's position, the variance in the event's position-corrected time of the PMT's discriminator firings, and the variance in the fit to the angular distribution of the observed light assuming the light is generated by an electron. Figure 8 shows the ID signals for $180 v_{\mu}+{ }^{12} \mathrm{C} \rightarrow \mu^{-}+X$ events with subsequent $\mu^{-} \rightarrow e^{-} v_{\mu} v_{e}$ decay. The first of these pairs of signals is the muon followed by the electron. The electron ID signal is seen to be well separated from the muon ID.

Neutron identification is carried out by the detection of the 2.2-MeV $\gamma$ ray that follows neutron thermalization and capture on the hydrogen $\left(\mathrm{CH}_{2}\right)$ in the detector. The rate of all events between 18 and 50 hit tubes is $1 \mathrm{KH}$ or about $6 \mathrm{H} / \mathrm{m}^{3}$.

The mean time for neutron capture in LSND is calculated to be $186 \mu \mathrm{s}$, dominated by the capture time on hydrogen $[n+p \rightarrow d+\gamma(2.22 \mathrm{MeV})]$. Figure 9a shows the spatial separation between the $\gamma$ event and the earlier event, the unshaded region of the figure shows the spatial correlation for events occurring between 0 $300 \mu \mathrm{s}$, while the shaded region shows the events occurring between 700-1000 $\mu \mathrm{s}$. The correlation in the earlier time bin is manifestly evident. The difference in the distribution of the number of hit tubes (Fig. 9b) over these two intervals is also manifestly clear, the unshaded area being what one expects for 2.2-MeV $\gamma$ rays, while the shaded spectrum is typical of the background in the tank. Figure $9 \mathrm{c}$ shows the time correlation between the triggering event and the subsequent $\gamma$ ray. The time correlation shows the expected mean time to

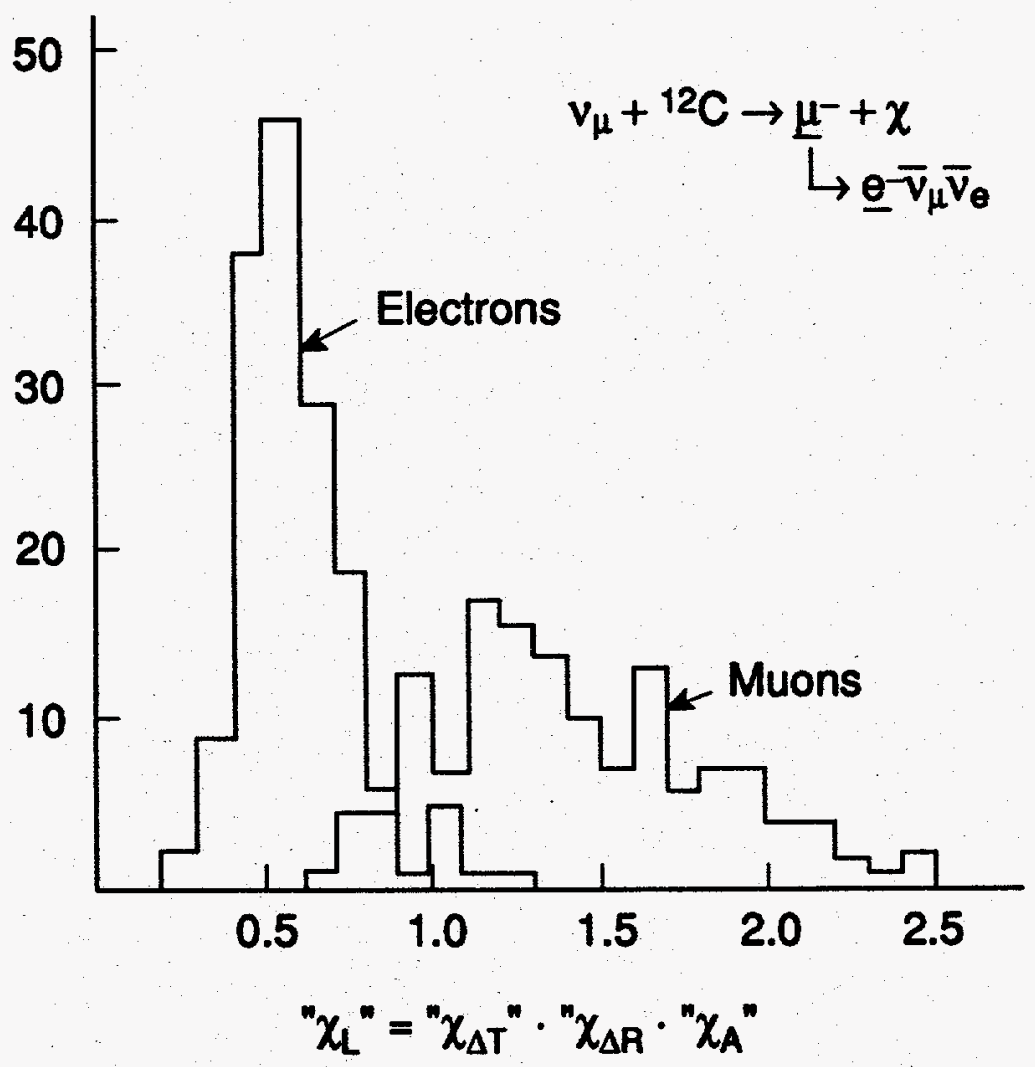

Fig. 8. Plot of the particle identification signals for muons and electrons from a sample of $v_{\mu}+{ }^{12} \mathrm{C} \rightarrow \mu^{-}+{ }^{12} \mathrm{~N}$ events. 

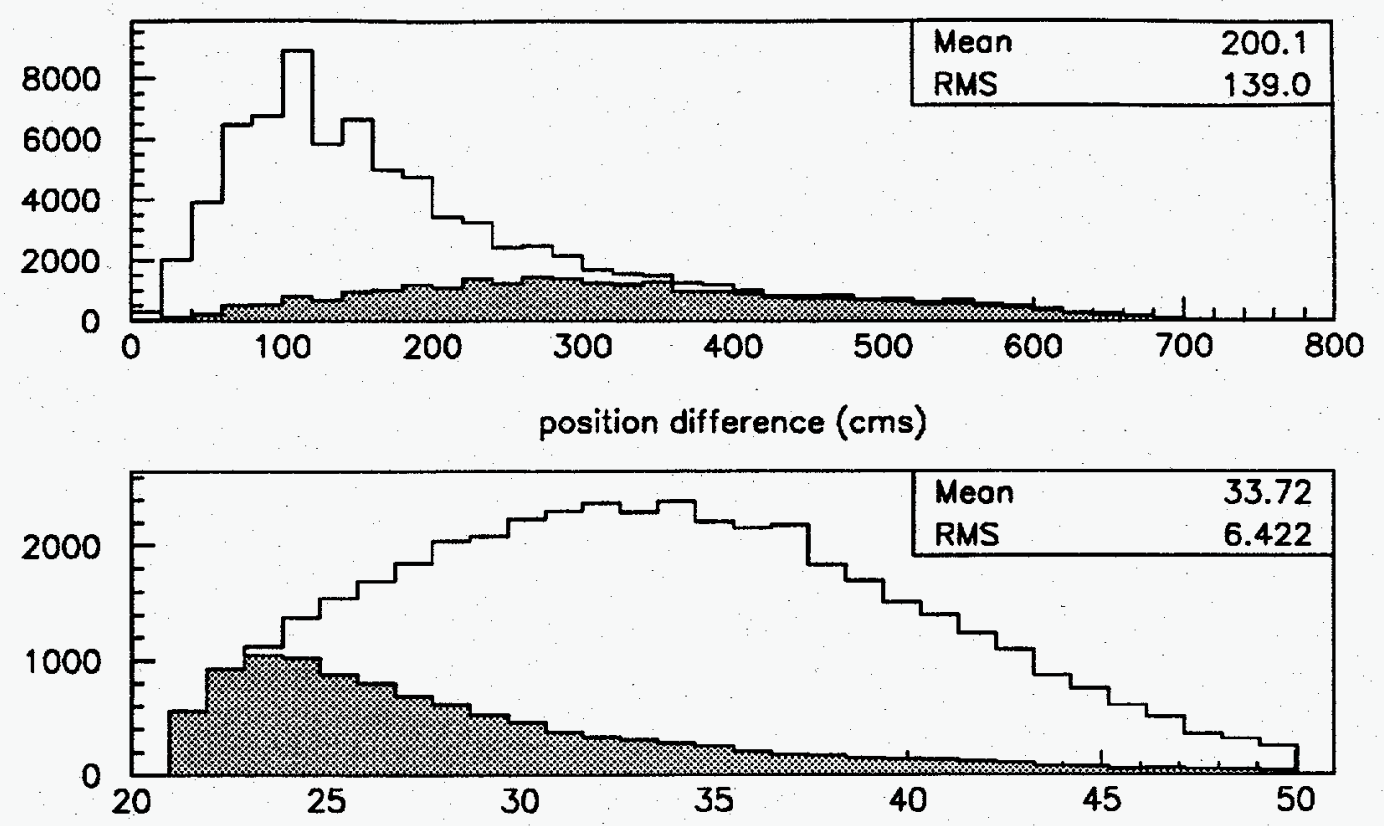

2.2 Mev gamma multiplicity

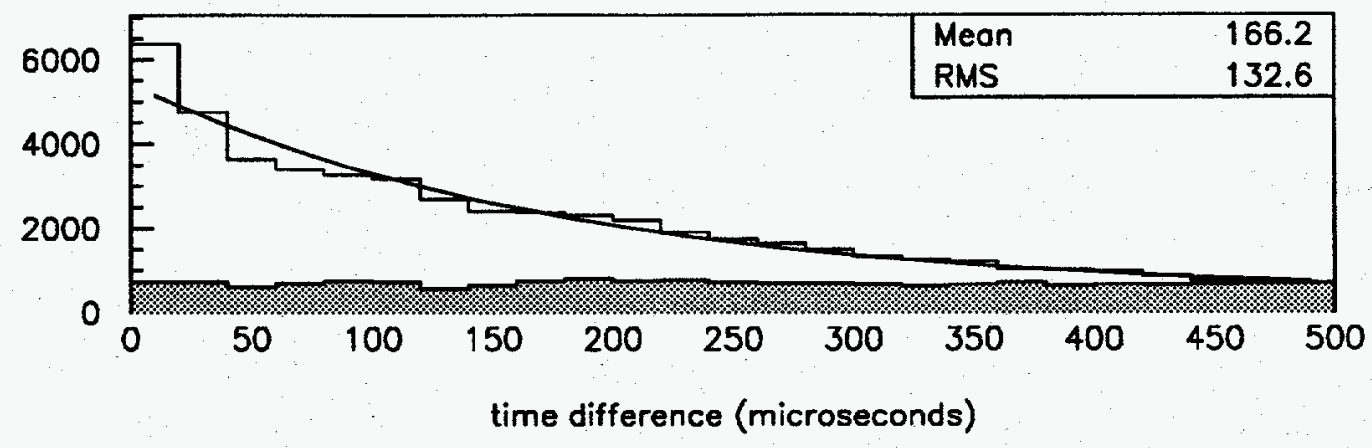

Fig. 9. (a) The separation between the position of the initial event and a subsequent gamma ray. (b) The number of hit tubes for events in the first $300 \mu$ s (unshaded) and events in the interval 700-1000 $\mu \mathrm{s}$. (c) Time interval between the triggering event and a $\gamma$ ray within $250 \mathrm{~cm}$.

capture. Our efficiency for detecting neutrino via the capture $\gamma$ rays is in excess of $75 \%$ with a less than $10 \%$ accidental background.

Thus, the identification of $e, \mu, p$, and $n$ is carried out as follows. Electrons are uniquely identified by having a particle ID signal $0.2 \leq x_{L} \leq 0.8$. Muons are identified via $0.8 \leq x_{L} \leq 2.5$ with a subsequent electron decay (small corrections need to be applied for the small fraction of $\mu^{-}$that capture on ${ }^{12} \mathrm{C}$ ). Protons have $0.8 \leq$ $x_{L} \leq 3.0$ with no subsequent radiation. Neutrons have $1 \leq x_{L} \leq 3.5$ and are separated by a $2.2-\mathrm{MeV} \gamma$ ray within $250 \mathrm{~cm}$ and $500 \mu \mathrm{s}$. A variety of other cuts are applied to eliminate cosmic-ray background.

Because the CR background is so large, it is necessary to subtract the "beam off" signal from the "beam on" signal to establish the beam excess. No gates are used in this process, rather a bit is set when the beamsensing pick off records protons exiting the accelerator. The beam-on to beam-off ratio is fixed via the number of tagged to untagged events of cosmic-ray origin. 
In the initial run of LSND in October 1993, a total of $214.5 \pm 85$ beam excess neutrons plus protons with energies above $36 \mathrm{MeV}$ were observed. The number expected was 239 . Above $60 \mathrm{MeV} 44 \pm 24$ proton (566 predicted) and $26.5 \pm 28$ neutrons (7 predicted) were found. The spectra shown in Fig. 10a and b are in agreement with expectation. A great deal more data will be accumulated on the August 11-November 30 run of this year due to longer running time, higher beam intensity, and lower thresholds.

It appears that measuring the axial vector form factor in low-energy $v$-nucleon elastic scattering is an excellent way to probe the spin structure of the nucleon. While experimentally difficult, it does not suffer from the problems that beset interpreting spin structure functions (extrapolation to $x=0$, higher twist correction, assumption of $\mathrm{SU}_{3}$ flavor symmetry). The early results from the LSND are encouraging but do not allow an estimation of the eventual accuracy to which $G_{s}(0)$ will be determined with the $94-95$ runs at LAMPF.

\section{HIGH-ENERGY $v \cdot N$ SCATTERING - AGS E734}

Before concluding, I want to call your attention to the recent reanalysis [1] of the currently best experiment [3] on high-energy $\left(E_{v}>1 \mathrm{GeV}\right)$ neutrino-nucleon elastic scattering. Using the results of earlier analysis, several authors [4,5] had cited AGS-E734 as supporting the EMC value for $G_{s}(0)$. However, that is too strong a statement. Using the formalism indicated in Eqs. (12)-(14), along with a standard parameterization of the known form factors, E734 was reanalyzed [11]. In the original analysis [3], E734 used the then world average for $M_{A}\left(1.032 \pm 0.036 \mathrm{MeV} / c^{2}\right)$ in the isovector axial form factor, while yielded $G_{s}=-0.12 \pm 0.07$. However, in a subsequent publication [15], E734 reported a new value $M_{A}=1.09 \pm 0.03 \pm 0.02 \mathrm{MeV} / \mathrm{c}^{2}$, which raised the world average to its present value of $M_{A}=1.061 \pm 0.026$. As shown in Table I (from Ref. [11]), the data can readily be fit within the new world average for $M_{A}$ with all strange form factors set equal to zero. A lower value of $\chi^{2}$ can be achieved by allowing $M_{A}$ to drop to $1.049 \mathrm{GeV}$, whereupon $G_{s}(0)=-0.15 \pm 0.07$. However, it is clear that little progress can be made in the determination of $G_{s}\left(Q^{2}\right)$, $Q^{2}>0.5 \mathrm{GeV}^{2}$ unless $M_{A}$ is more precisely determined. This appears possible to do in a new dedicated experiment, which would have to obtain both precise charged and neutral current data.

Table I. The fit results for the strange form factors $G_{1}^{s}(0)=\Delta s, F_{1}^{s}=-(1 / 6)\left\langle r_{s}^{2}\right\rangle$, and $F_{2}^{s}(0)=\mu_{s}$, and the axial-vector dipole mass $M_{A}$ (from Ref. [14]).

\begin{tabular}{lccccc}
\hline Fit & $G_{1}^{2}(0)$ & $F_{1}^{s}$ & $F_{2}^{s}(0)$ & $M_{A}$ & $\chi^{2} / N_{\text {DOF }}$ \\
\hline I & 0 & 0 & 0 & $1.086 \pm 0.015$ & $14.12 / 14$ \\
II & $-0.15 \pm 0.07$ & 0 & 0 & $1.049 \pm 0.019$ & $9.73 / 13$ \\
III & $-0.13 \pm 0.09$ & $0.49 \pm 0.70$ & $-0.39 \pm 0.70$ & $1.049 \pm 0.023$ & $9.28 / 11$ \\
IV & $-0.21 \pm 0.10$ & $0.53 \pm 0.70$ & $-0.40 \pm 0.72$ & $1.012 \pm 0.032$ & $8.13 / 11$ \\
\hline
\end{tabular}

The E734 data do, however, an excellent job of fixing the sum of the strange vector form factors $F_{1}\left(Q^{2}\right)+F_{2}\left(Q^{2}\right)$ at $Q^{2}=0.75 \mathrm{GeV} / c^{2}$. This is because E734 obtained $d \sigma / d Q^{2}$ for both $v p$ and $\bar{v} p$, and the difference in these cross sections is governed by the interference term appearing in Eq. (14). Figure 11 shows the values of $G_{M}\left(Q^{2}\right)$ and $G_{E}\left(Q^{2}\right)$ extracted from the analysis of Ref. [11], along with some of the theoretical predictions for these quantities. 

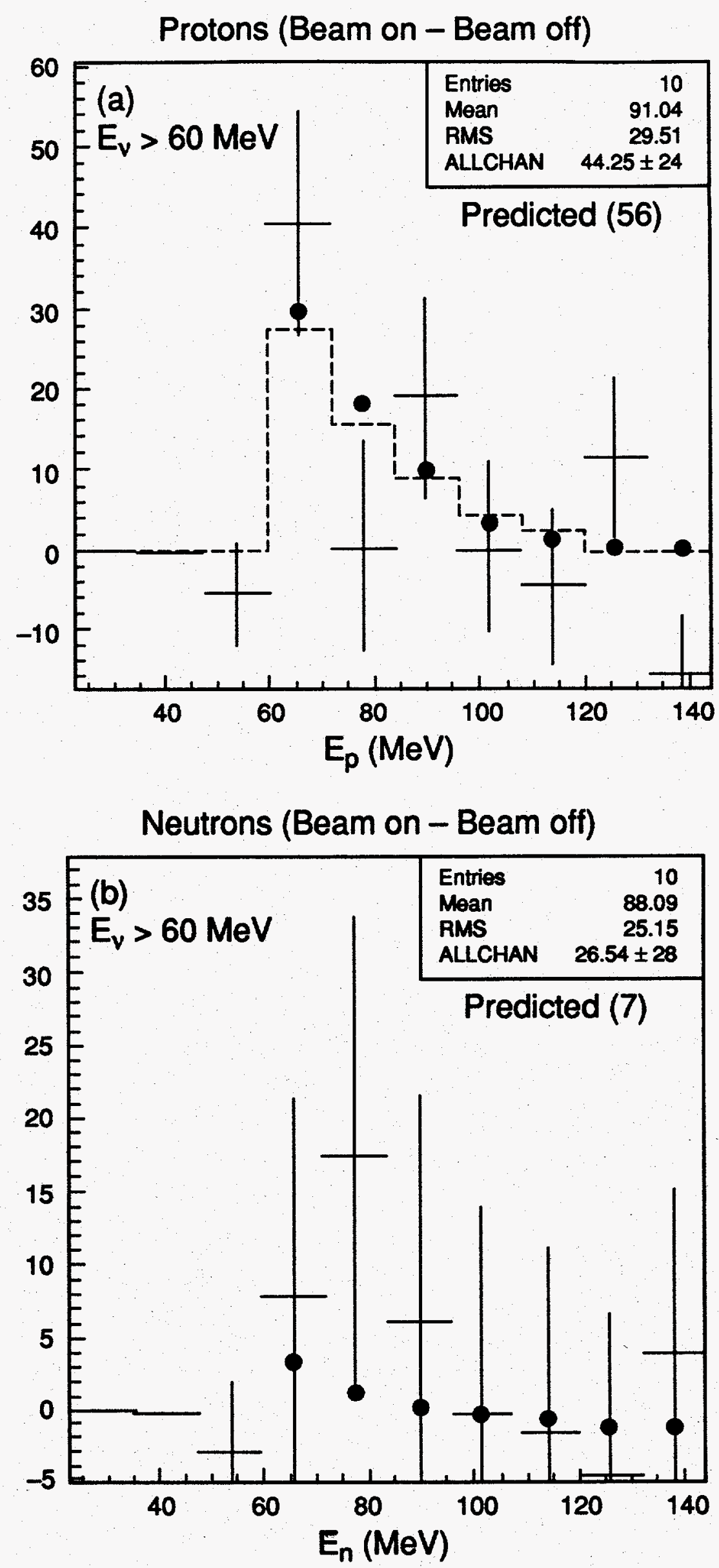

Fig. 10. (a) Beam excess of protons. (b) Beam excess of neutrons. 

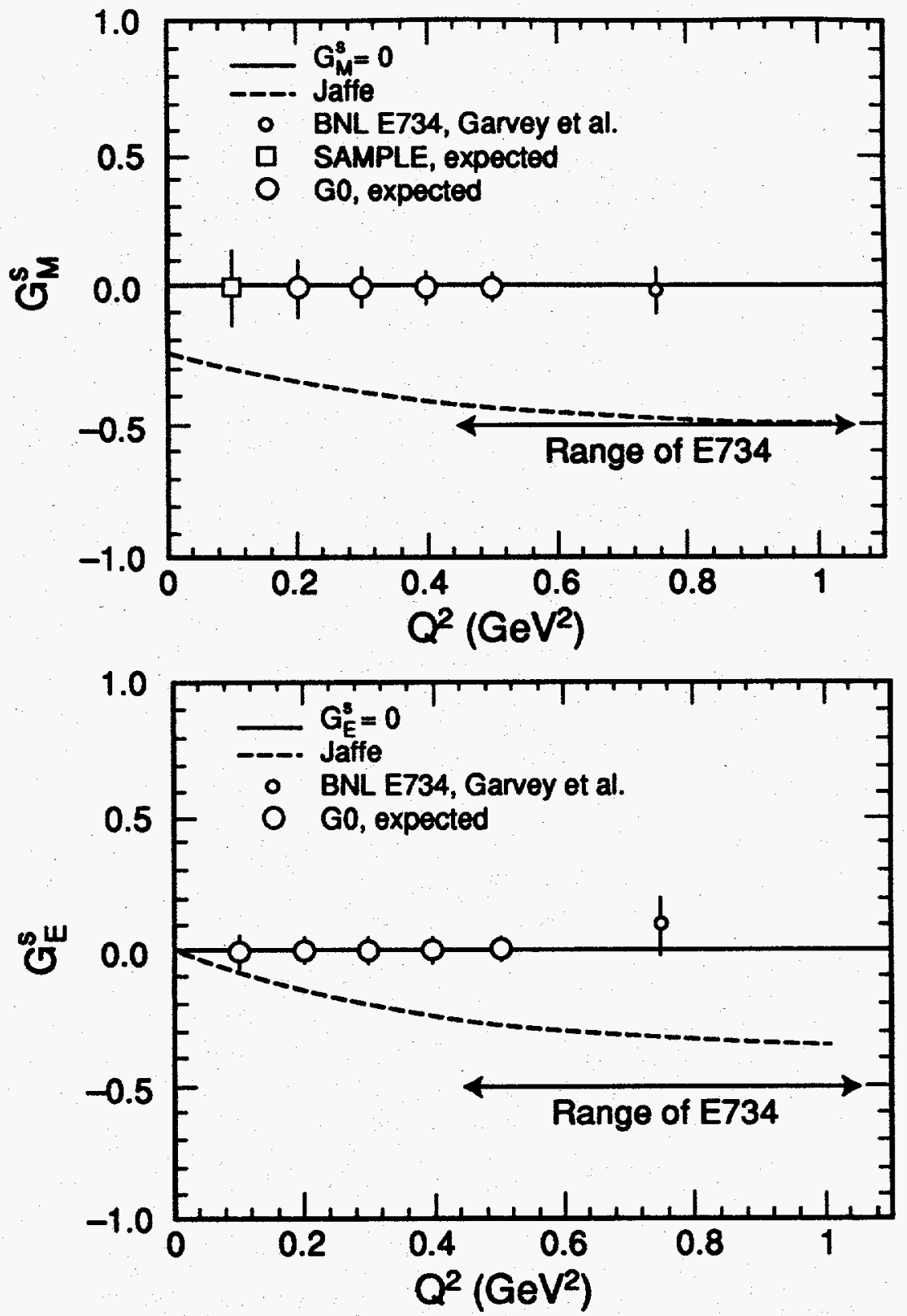

Fig. 11. The value of $G_{M}\left(Q^{2}\right)=F_{1}\left(Q^{2}\right)+F_{2}\left(Q^{2}\right)$ at $Q^{2}=0.75 \mathrm{GeV} / c^{2}$ extracted from E734 (AGS). The other points are projected from approved experiments employing parityviolating $\vec{e}, e$ scattering. The central values of these projections use predictions [16] for the strange vector form factors. The figure is from Ref. [17].

\section{REFERENCES}

[1] Ashman, J. et al. (1988). Phys. Lett. 206B, 364; (1989). Nucl. Phys. B 328, 1.

[2] LSND - LAMPF Experiment 1173 (unpublished).

[3] Ahrens, L. A. et al. (1982). Phys. Rev. D 34, 785.

[4] Kaplan, D. and A. Manohar (1988). Nucl. Phys. B 310, 527.

[5] Ellis, J. and M. Karliner (1988). Phys. Lett. 213B, 73.

[6] Collins, J., F. Wilczek and A. Zee (1978), Phys. Rev. D 18, 242.

[7] Mohapatra, R. N. and G. Senjanovic (1979), Phys. Rev. D 19, 2165.

[8] Wolfenstein, L. (1979). Phys. Rev. D 19, 3450. 
[9] Llewellyn-Smith, C. H. (1972). Phys. Rep. 3C, 261.

[10] Beise, E. and R. McKeown (1991). Comm. Nucl. Part. Phys. 20, 105.

[11] Garvey, G. T., W. Louis and H. White (1993). Phys. Rev. C 48, 761.

[12] Garvey, G. T. et al. (1992). Phys. Lett. 289B, 249; (1993). Phys. Rev. C 48, 1919.

[13] Horowitz, C. J. et al. (1993). Phys. Rev. C 48, 3076.

[14] Reeder, R. A. et al. (1993). Nucl. Instrum. Methods A 334, 353.

[15] Ahrens, L. A. et al. (1988). Phys. Lett. 202B, 284.

[16] Jaffe, R. L. (1989). Phys. Lett. 229B, 275.

[17] Boested, P. et al. (1993). $G_{0}$ Technical Design Report (unpublished).

\section{DISCLAIMER}

This report was prepared as an account of work sponsored by an agency of the United States Government. Neither the United States Government nor any agency thereof, nor any of their employees, makes any warranty, express or implied, or assumes any legal liability or responsibility for the accuracy, completeness, or usefulness of any information, apparatus, product, or process disclosed, or represents that its use would not infringe privately owned rights. Reference herein to any specific commercial product, process, or service by trade name, trademark, manufacturer, or otherwise does not necessarily constitute or imply its endorsement, recommanufacturer, or othion, or favoring by the United States Government or any agency thereof. The views and opinions of authors expressed herein do not necessarily state or reflect those of the United States Government or any agency thereof. 